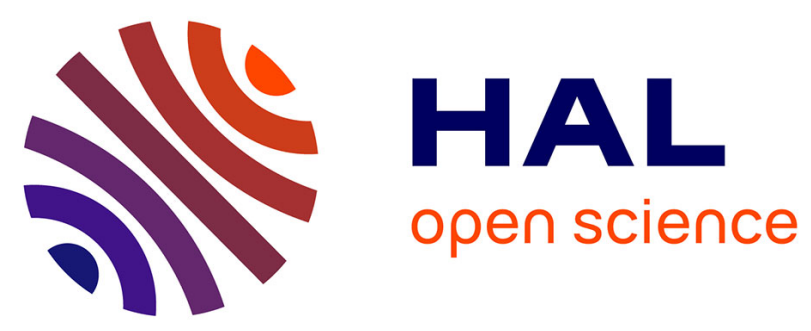

\title{
Wetting film dynamics during evaporation under weightlessness in a near-critical fluid
}

\author{
John Hegseth, Ana Oprisan, Yves Garrabos, Vadim Nikolayev, Carole
} Lecoutre-Chabot, Daniel Beysens

\section{To cite this version:}

John Hegseth, Ana Oprisan, Yves Garrabos, Vadim Nikolayev, Carole Lecoutre-Chabot, et al.. Wetting film dynamics during evaporation under weightlessness in a near-critical fluid. Physical Review E: Statistical, Nonlinear, and Soft Matter Physics, 2005, 72 (3), 031602 (12 p.). 10.1103/PhysRevE.72.031602 . hal-01261762

\section{HAL Id: hal-01261762 \\ https://hal.science/hal-01261762}

Submitted on 26 Jan 2016

HAL is a multi-disciplinary open access archive for the deposit and dissemination of scientific research documents, whether they are published or not. The documents may come from teaching and research institutions in France or abroad, or from public or private research centers.
L'archive ouverte pluridisciplinaire HAL, est destinée au dépôt et à la diffusion de documents scientifiques de niveau recherche, publiés ou non, émanant des établissements d'enseignement et de recherche français ou étrangers, des laboratoires publics ou privés.

\section{(ㅇ)(1) $\$$}

Distributed under a Creative Commons Attribution - NonCommercial - NoDerivatives| 4.0 


\title{
Wetting film dynamics during evaporation under weightlessness in a near-critical fluid
}

\author{
John Hegseth and Ana Oprisan \\ Department of Physics, University of New Orleans, New Orleans, Louisiana 70148, USA \\ Yves Garrabos, Vadim S. Nikolayev, Carole Lecoutre-Chabot, and Daniel Beysens \\ ESEME, Institut de Chimie de la Matière Condensée de Bordeaux, CNRS, Université de Bordeaux I, Avenue du Dr. Schweitzer, \\ F-33608 Pessac Cedex, France \\ (Received 3 May 2004; revised manuscript received 18 May 2005; published 12 September 2005)
}

\begin{abstract}
By performing near-critical fluid experiments in the weightlessness of an orbiting space vehicle, we have suppressed buoyancy-driven flows and gravitational constraints on the liquid-gas interface of a large gas bubble. At equilibrium, the liquid completely wets the walls of a cylindrical cell, and the bubble is pushed to the sidewall. In these experiments the system's temperature $T$ is increased at a constant rate past the critical temperature $T_{C}$, pushing it slightly out of equilibrium. The wetting film shows a large mechanical response to this heating, including contact lines that recede on a solid surface and a spreading bubble. Near $T_{C}$, the receding contact lines make the entire bubble appear to spread along the copper sidewall. The spreading bubble is a manifestation of the boiling crisis near the critical point. We present quantitative data of the receding contact lines that are observed prior to the near-critical boiling crisis. We analyze the receding contact lines in detail, and find that they are driven by vapor recoil from evaporation, as is the spreading bubble of the boiling crisis.
\end{abstract}

DOI: 10.1103/PhysRevE.72.031602

PACS number(s): 68.03.Cd, 05.70.Np, 44.35.+c

\section{INTRODUCTION}

When a pure fluid is boiled, many complex dynamical processes occur simultaneously. These include heat transport, convection, phase changes, and interfacial processes [1]. The buoyancy force causes many complications, i.e., buoyancy lifts gas bubbles that nucleate on a hot surface and leads to the agitation of the fluid, making both observation and modeling difficult. In the weightless environment of an orbiting spacecraft, however, the buoyancy force is negligible. Much of the complex fluid dynamics is suppressed, and the observation of the interfacial processes is facilitated. Material and thermal properties also play an important role in the boiling process. The properties include surface tension, solid surface wetting, liquid-gas density differences, and thermal diffusivity. Near the liquid-gas critical point these properties vary with temperature according to well-known universal power laws that either converge to zero or diverge (e.g., the surface tension goes to zero) [2]. These properties lead to perfect wetting by the liquid phase (zero contact angle) near the critical temperature $T_{C}$ [3]. When heat is applied to push the system slightly out of equilibrium, we expect a perfectly wetted wall to dry from evaporation, resulting in liquid-gassolid contact lines. The same physics that makes perfect wetting at equilibrium will result in a zero contact-angle boundary condition when a contact line appears.

In the following we report on the behavior of a large gas bubble inside a thin constant mass cell when heat is applied. The cell is filled with fluid very close to the liquid-gas critical density. The thin cell produces a considerable constraint on the bubble's shape and allows the entire bubble to be observed as the temperature increases. We recorded vigorous contact line motion below the coexistence temperature $T_{\mathrm{CX}}$. A spectacular spreading bubble process occurs near the critical temperature $T_{C}$, where the gas bubble changes shape to cover almost half the cell's copper sidewall (note that $T_{C}=T_{\mathrm{CX}} \pm 50 \mu \mathrm{K}$ for all of the densities in these experiments) $[4,5]$. Both the spreading bubble and the contact line motion occur when the bubble is initially in contact with a highly conductive copper sidewall.

The large heat transfer that boiling facilitates has led to many types of heat transfer technology and many empirical models of limited validity. This spreading bubble behavior has been identified with the covering of a heater with a gas film also known as the "boiling crisis" in the theory of boiling $[1,4]$. In practical applications the boiling crisis usually results in a catastrophic failure of the heat transfer device. A mechanism for this spreading is clearly revealed near the critical point [4], i.e., the vapor recoil from evaporating fluid pushes the liquid near the liquid-gas-solid contact line. Such an effect also occurs during conventional boiling at a particular heat flux called the critical heat flux. The spreading bubble is seen near the critical point without a large heat flux because the critical heat flux is much smaller near the critical point [4].

\section{APPARATUS AND OPTICAL TECHNIQUE}

These experimental results were obtained in the weightlessness of an orbiting spacecraft (MIR space station) on the French/American GMSF mission using the Alice-II instrument $[4,5]$. This instrument is specially designed to obtain high-precision temperature control (stability of $\approx 15 \mu \mathrm{K}$ over $50 \mathrm{~h}$, repeatability of $\approx 40 \mu \mathrm{K}$ over 7 days). To place the samples near the critical point, constant mass cells are prepared with a high-precision density (to $0.02 \%$ ) by observing the volume fraction change of the cells as a function of temperature on the ground [6]. Similar ground-based experiments were done before these experiments yielding com- 


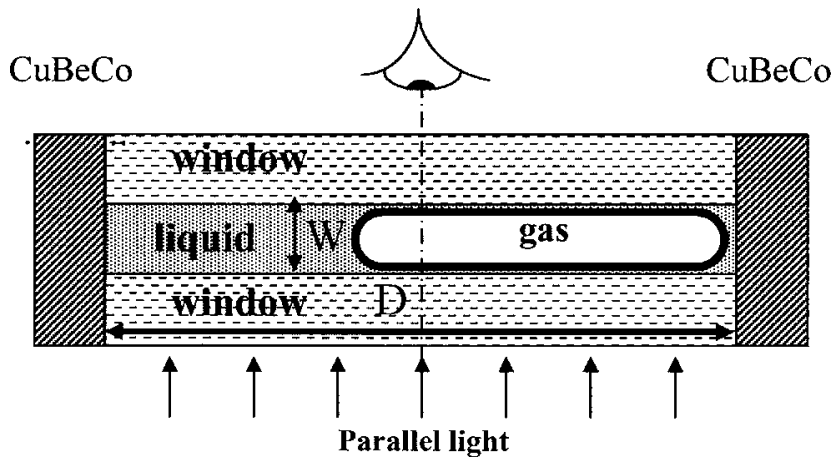

FIG. 1. A cross-section view of the cylindrical sample cell. The fluid volume is contained between two sapphire windows and a $\mathrm{CuBeCo}$ alloy ring (sidewall). The dimensions $(W, D)$ of the cell are indicated. The semicircular liquid-gas meniscus between the two parallel windows is shown. This interface appears dark in the images because the liquid-gas meniscus refracts the normally incident parallel light away from the cell axis.

pletely different results in the presence of gravity [7]. In this case the interface is horizontal except very near a wall. When the cell windows are also horizontal, the heating results in drops that form at the wetted top of the cell in what appears to be a Rayleigh-Taylor instability. These drops form at the top window and fall continuously in the cell.

In these experiments, a thin layer of $\mathrm{SF}_{6}$ or $\mathrm{CO}_{2}$ of width $W$ was sandwiched between two sapphire windows and surrounded by a copper housing in the optical cell shown in Fig. 1. Two $9.0 \mathrm{~mm}$ long cylindrical sapphire windows of diameter $D=12 \mathrm{~mm}$ are pressed into a copper block with a corre-
TABLE I. The three experiments A, B, and $\mathrm{C}$ used three different cells. Cells A and B were filled with $\mathrm{SF}_{6}$, and cell $\mathrm{C}$ was filled with $\mathrm{CO}_{2}$. These three cylindrical cells had different widths $W$ and were filled to different densities, as shown in the table. Definitions of $W, D$, and $\Gamma$ are given in the text and in Fig. 1. Experiment $\mathrm{C}$ was performed in a variable volume cell.

\begin{tabular}{llll}
\hline \hline Experiment & Fluid & $\left(\rho-\rho_{c}\right) / \rho_{c}$ & $W(m m), \Gamma=D / W$ \\
\hline A & $\mathrm{SF}_{6}$ & $0.85 \%$ & $3.016,3.979$ \\
$\mathrm{~B}$ & $\mathrm{SF}_{6}$ & $0.25 \%$ & $1.664,7.211$ \\
$\mathrm{C}$ & $\mathrm{CO}_{2}$ & $-3.5 \%$ & $2.204,5.445$ \\
\hline \hline
\end{tabular}

sponding cylindrical hole and glued to the copper at the sides of the sapphire. This method avoids the unknown volume associated with O-rings, etc., and allows the above highprecision density measurements to be verified. Three cells were used to perform these experiments, and Table I summarizes their key features. A two-phase liquid-gas mixture consisting of a single gas bubble surrounded by liquid [see Fig. 2(b)] passed near the critical temperature as it was heated to a supercritical fluid. In experiments A and B the bubble was slightly pushed against the sidewall of the cell, and in experiment $\mathrm{C}$ it did not touch the sidewall. The $\mathrm{CO}_{2}$ cell in experiment $\mathrm{C}$ contained three thermistors that make local temperature measurements in the fluid. Typically one thermistor was in the gas while the others were in the liquid. We will discuss the results of these measurements elsewhere. In addition, this cell has a sealed piston that can slide in and out of the cell volume to change the fluid's average density. These objects prevented the bubble from touching the copper sidewall as it

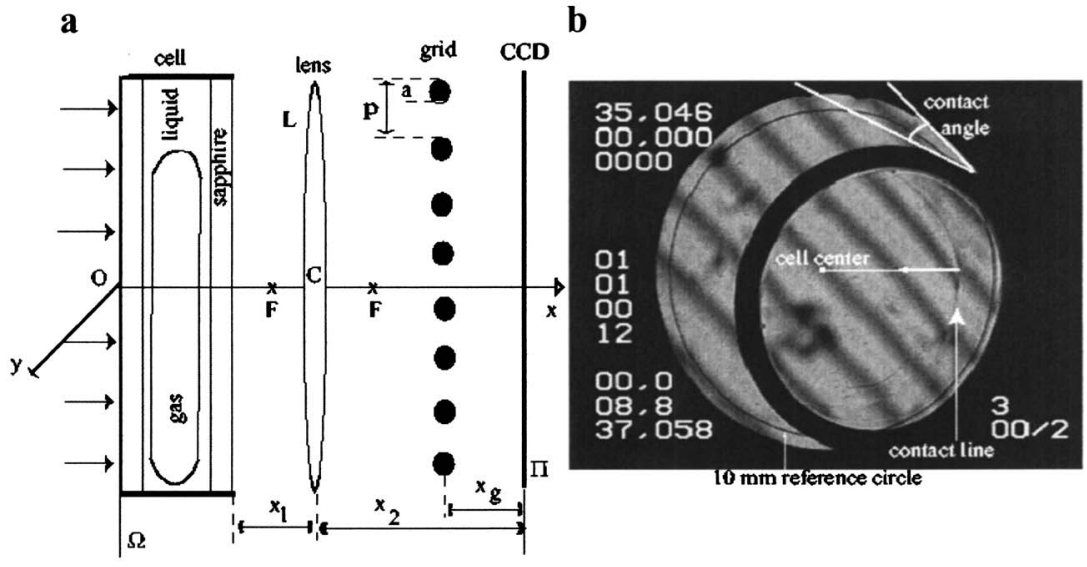

FIG. 2. (a) The cell (from experiment B) is a $D=12 \mathrm{~mm}$ diameter cylinder made of $\mathrm{CuBeCo}$ containing a $W=1.664 \mathrm{~mm}$ layer of $\mathrm{SF}_{6}$ sandwiched between two sapphire windows ( $9 \mathrm{~mm}$ thick). The convergent lens $L$, with focal point $F$ and the center $C$, projects the out-of-focus grid image on the CCD plane $\Pi$ [panel (b)]. This system can detect a density change of up to $7.8 \times 10^{-5} \mathrm{~g} / \mathrm{cm}^{3} / \mathrm{pixel}$. The density may then be found by integrating the local density changes from a given reference density. This technique is sufficient to detect steep density gradients in the fluid. It is only capable, however, of detecting $4.3 \%$ constant density gradient change across $1 \mathrm{~cm}$ length of the cell of $\mathrm{SF}_{6}$. In a liquid-gas mixture, these displacements may be analyzed using the paraxial approximation of geometrical optics. (b) Shown is the defocused grid image recorded by the CCD camera. The evaporation of the liquid film between the sapphire window(s) and the gas bubble leads to a gas-liquid-solid contact line. The part of the grid shadows that is not distorted is to the right of the contact line, indicating the existence of a dry gas-solid interface. When heating is first applied, a receding contact line and a simultaneous change in the bubble/ sidewall contact angle, shown above, are seen. The heat transfer through the cell's copper sidewall evaporates the liquid film, causing this change in the bubble and/or sidewall contact angle. Also shown is the $10 \mathrm{~mm}$ diameter reference circle, the center of the cell, the reference line, and the point used in Fig. 9 and Fig. 10. 


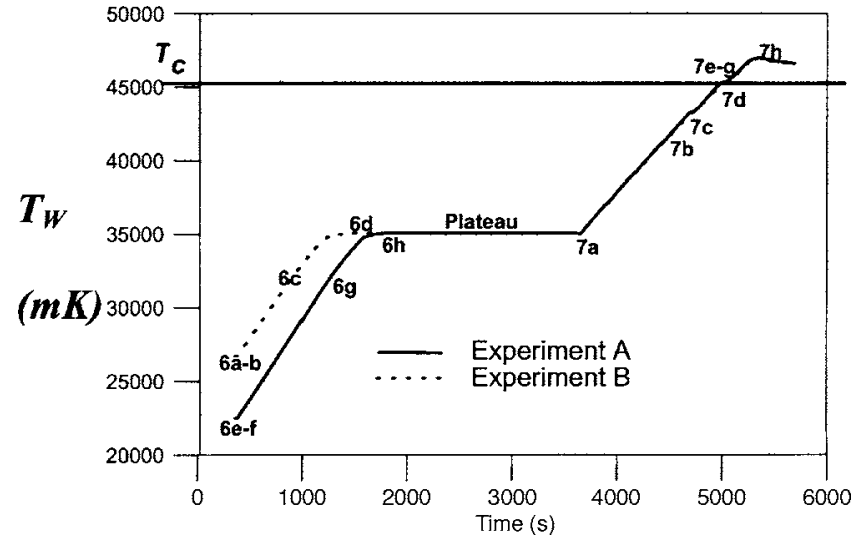

FIG. 3. Temperature ramp applied to cells A and B. The initial ramp has an $8.7 \mathrm{mK} / \mathrm{s}$ rate followed by a 40 min plateau and a $7.4 \mathrm{mK} / \mathrm{s}$ ramp. $T_{W}$ is the temperature measured in the copper sidewall. Also shown are the times and temperatures for the sequence of images in Figs. 6 and 7.

was heated. The two cells that were used in experiments A and $\mathrm{B}$ did not have a piston or thermistors allowing the bubble to move and touch the cell sidewall. These two cells only differed by $W$ or the aspect ratio $\Gamma(\Gamma=D / W$; see Table I). Two nearly identical runs were performed in each cell to observe how the geometry affects the spreading bubble.

Because the contact angle is zero near the critical point, the liquid-gas meniscus between the two parallel windows forms an interface with a semicircular cross section between the windows, as shown schematically in Fig. 1. This interface appears dark in the image [see Fig. 2(b)] because the liquid-gas meniscus refracts the normally incident light away from the cell axis. We have verified by ray tracing that all of the normally incident light on the meniscus is refracted out of the field of view. This shows that in normally incident light the minimum thickness of the dark region measures the radius of the meniscus cross section (a principal radius of curvature) [5]. We also note that some of the fluid volume near each cell's edge is not visible in our images, i.e., the light at the edge of each cell is blocked by glue that was used in manufacturing the cells. This radial edge distance is approximately $1 \mathrm{~mm}$. Nevertheless, we can find each cell's center and each cell's edge because there is a $10 \mathrm{~mm}$ diameter reference etching on the sapphire windows that is concentric with the cell [see Fig. 2(b)].

Each cell's temperature is linearly increased in time to a temperature greater than $T_{\mathrm{CX}}\left(\approx 45.5^{\circ} \mathrm{C}\right.$ for $\mathrm{SF}_{6}$ and $\approx 31.2{ }^{\circ} \mathrm{C}$ for $\mathrm{CO}_{2}$ ), while the liquid-gas interface was visualized through light transmission normal to the windows. The temperature profile for the $\mathrm{SF}_{6}$ cells consisted of two ramps with a constant temperature plateau between, as shown in Fig. 3. An initial heating ramp at a rate of $8.7 \mathrm{mK} / \mathrm{s}$ is followed by approximately $40 \mathrm{~min}$ of constant temperature to allow the fluid to come into thermal equilibrium and produce a consistent initial condition. A second ramp through $T_{\mathrm{CX}}$ at a rate of $7.4 \mathrm{mK} / \mathrm{s}$ is then imposed and the critical point is crossed. The temperature ramp for experiment $\mathrm{C}$ was very similar. Identical commands were given to our instrument (ALICE II) for experiments A and B, respectively. The imposed ramps that resulted as checked by comparing corresponding temperature data were identical to within $40 \mathrm{mK}$.

The density changes in this highly compressible fluid are visualized by using a defocused grid (or grid-shadow) technique [8]. As discussed below, this technique also allows thickness changes in the liquid wetting film to be visualized, as shown in Fig. 4. Figure 2 shows how the defocused grid is projected onto the plane of the CCD. A plane wave propagating in the $x$ direction illuminates the cell filled with fluid $(\Omega)$. An image of $\Omega$ is projected onto a plane $\Pi$ by a lens $L$. A grid made up of equally spaced straight wires is placed perpendicular to the optical axis $x$ and parallel to the $y$ axis between $L$ and $\Pi$ [9]. The image of the grid is recorded at the plane $\Pi$ by the CCD camera. The magnification of the optical system is $\gamma=x_{2} / x_{1}$, where $x_{2}$ and $x_{1}$ are shown in Fig. 2 . If the liquid-gas interface is flat [see Fig. 2(a)] then the incident rays that are parallel to $x$ will remain parallel after they cross the cell. The resultant image of the grid shadow is not distorted.

As discussed below, we have observed receding contact lines on the sapphire windows. The principle of these measurements is the following. In the presence of any incline in the liquid-gas interface in the cell $\Omega$, the incident rays are

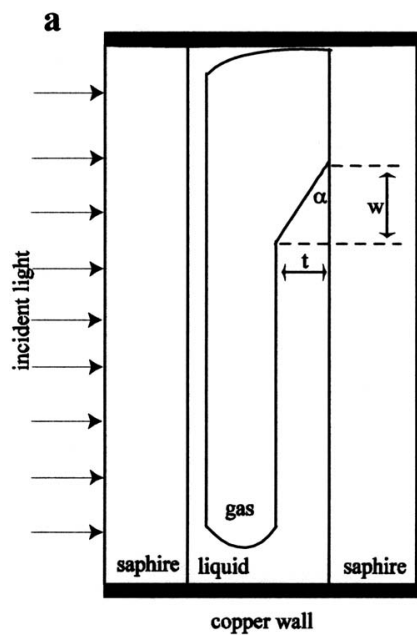

b

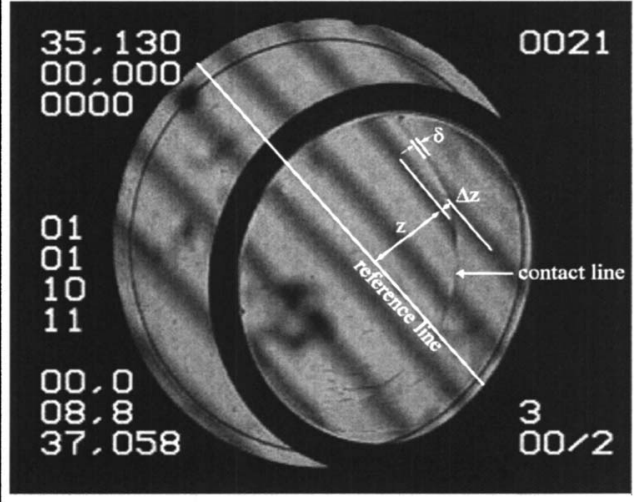

FIG. 4. (a) The tilted gas-liquid interface due to liquid evaporation leads to a contact line. (b) The tilt angle induces a significant displacement of the grid shadows. The shadow displacement depends on the tilt angle $\alpha$, the width $w$, and the thickness $t$ of the film (see the text for details). Also shown are $z, \Delta z, \delta$, and the reference line used in the calculations of the thickness $t$ of the film. 


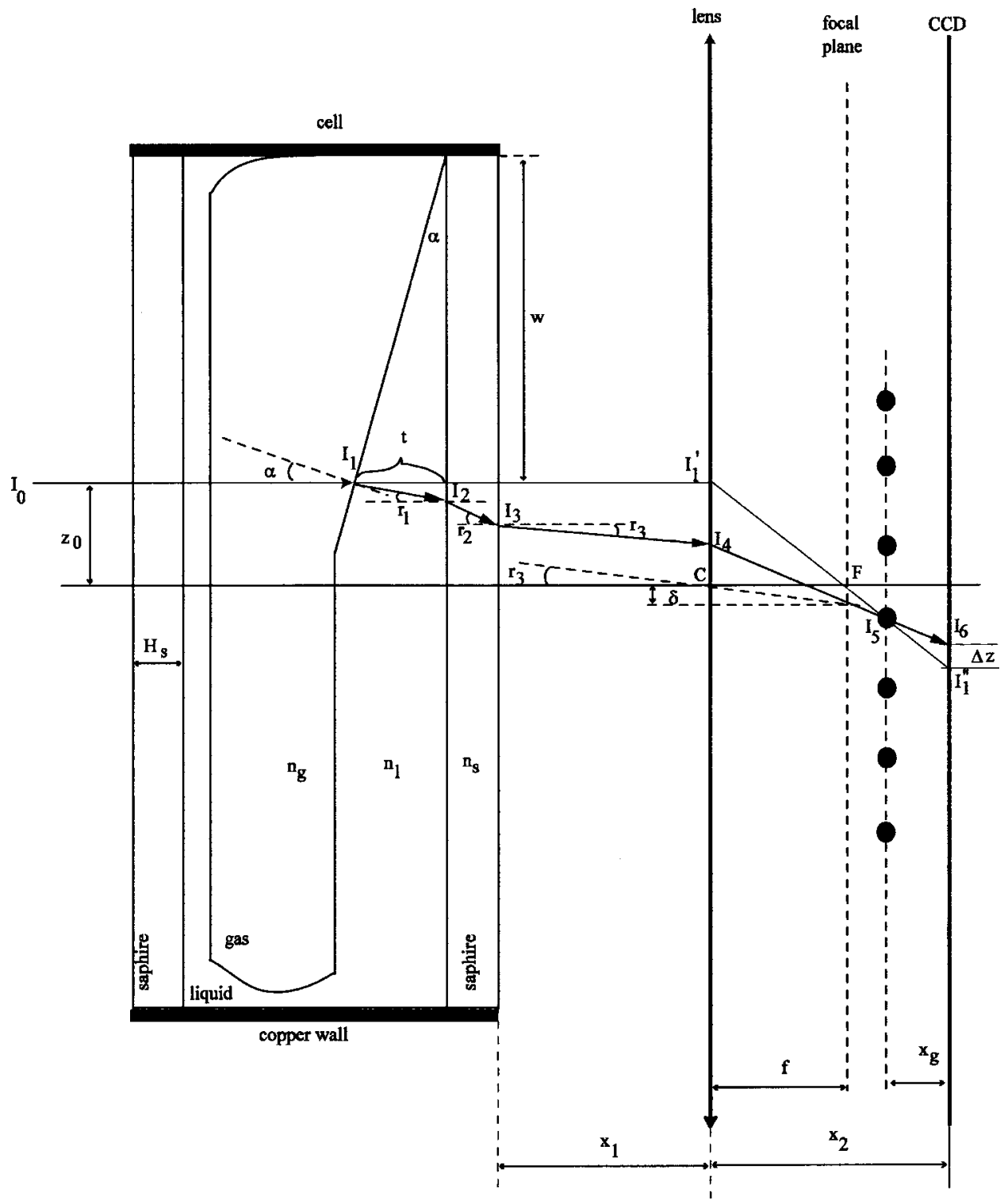

FIG. 5. Ray tracing through the cell and the optical system. The tilt angle of the gas-liquid interface is exaggerated for illustration purposes and is shown only on one side for simplicity. An incident ray $I_{0}$, passing at distance $z_{0}$ from the optical axes, would remain parallel to the optical axes if the interface is not tilted with respect to the sapphire windows, and would hit the CCD plane at $I_{1}^{\prime \prime}$. If there is a tilt angle $\alpha$ of the gas-liquid interface, then the ray starting at $I_{0}$ would hit the CCD plane at $I_{6}$ following successive refractions at $I_{1}, I_{2}, I_{3}$, and $I_{4}$. The displacement of the shadow $\Delta z$ depends on the tilt angle $\alpha$ of the interface and its thickness $t$.

deflected from their original direction along the $x$ axis. This causes a displacement and/or a distortion of the shadow of the grid on plane $\Pi$. The contact line shown in Fig. 4 is a typical localized line that appears in these experiments. This line is caused by the interface curvature. In fact, there should be two edges near the contact line. The wetting condition of the interface at the contact line requires a concave curvature near the wall, while the flat interface away from the contact line requires a convex curvature. Between the convex and concave edges is an inflection point and the entire variation of slope has a mean value. This suggests that between these edges and near the contact line a linear approximation is a reasonable simplification for the shape of the film profile. Using this wedge model, the shadow displacement depends on the effective angle $\alpha$ of the interface, as shown in Fig. 4.
If the liquid-gas interface is tilted $(\alpha \neq 0)$, as shown in Fig. 5, then a normal ray incident on the cell surface suffers successive refractions at the liquid-gas (Fig. 5, point $I_{1}$ ), liquid-sapphire (Fig. 5, point $I_{2}$ ), and sapphire-air (Fig. 5, point $I_{3}$ ) interfaces. Because of these successive refractions, the emergent ray leaves the cell at $I_{3}$ with an angle $r_{3} \neq 0$. Assuming that the refraction angle $r_{3}$ is small, the convergent lens will finally form the image on the CCD plane at $I_{6}$. In the example shown in Fig. 5, a flat liquid-gas interface would project the initial ray $I_{0}$ to the grid plane at $I_{5}$ and to $I_{1}^{\prime \prime}$. If the liquid-gas interface is tilted, then the same incident ray $I_{0}$ would pass through the same point $I_{5}$ in the grid plane and hit the CCD at $I_{6}$, distorting the image of the grid. We used a ray-tracing program that projects $10^{3}-10^{6}$ equally spaced incident rays into the cell parallel to $x$ [9]. For each incident 

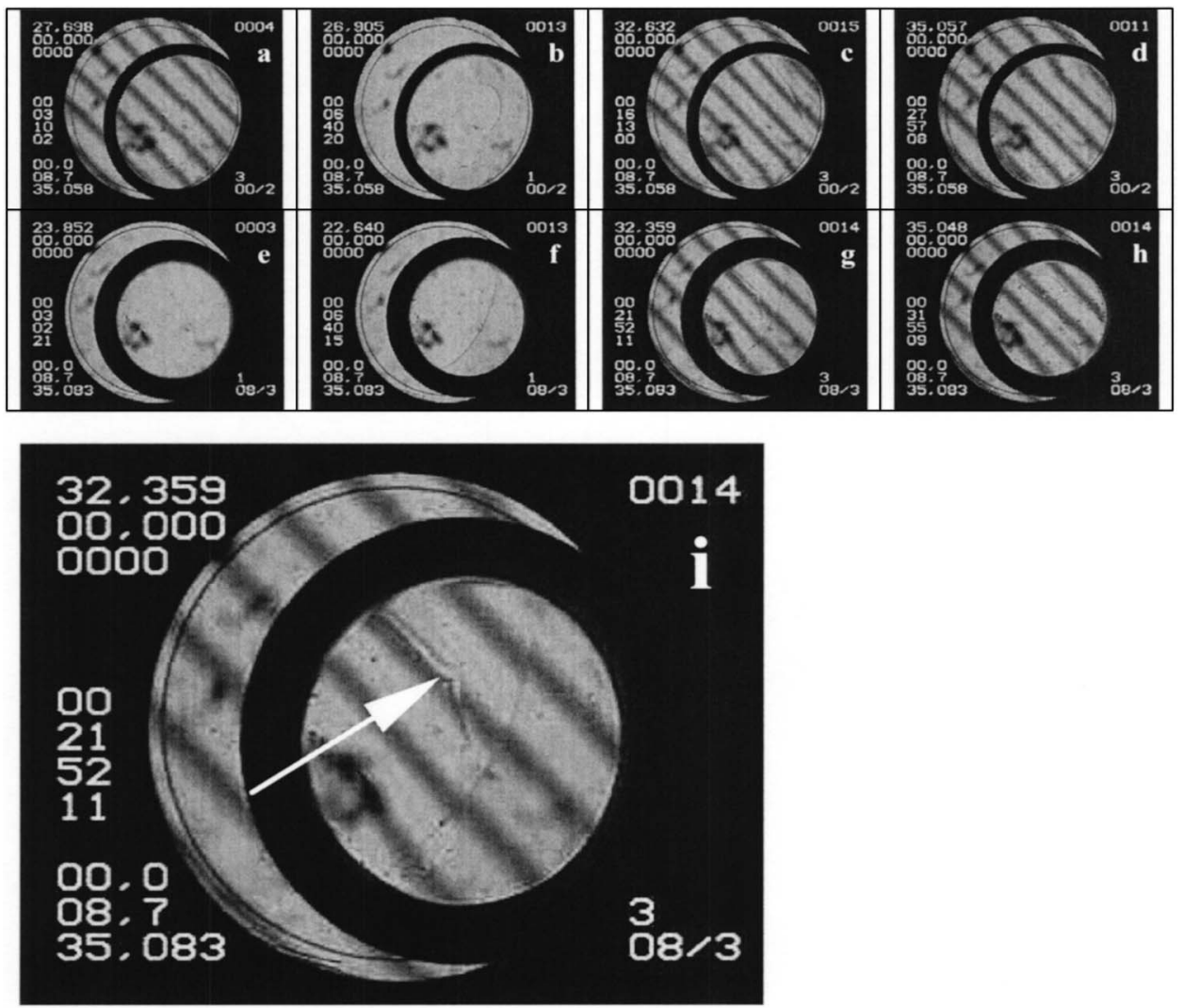

ray the position of the point of incidence on the CCD plane is computed to get the out-of-focus image of the grid by ray tracing.

The total displacement of the grid shadow $\Delta z(t, \alpha)$ depends on both the tilt angle $\alpha$ and the thickness of the interface $t$ by the formula (see the Appendix for derivation)

$$
\Delta z(t, \alpha)=-\left(t \tan \left(\alpha-r_{1}\right)+H_{s} \tan r_{2}\right) \gamma,
$$

where $r_{1}=r_{1}(\alpha)$ is the refracted angle at the wedge, $r_{2}$ $=r_{2}(\alpha)$ is the refracted angle at the liquid-sapphire interface, and $H_{s}$ is the thickness of the sapphire widow. By analyzing the image data shown in Figs. 4, 6(a), and 6(c), we can measure $\Delta z(t, \alpha)$ and the width of the triple contact line in the image plane $\Pi$. The line in the images of width $\delta$ is caused by a rapid change in film thickness that refracts the incident light producing the intensity change of the line. This rapid change in the contact line thickness is manifested by the discontinuity in the grid shadows, as can be seen at $\Delta z$ in Fig. 4(b). The width $\delta$ of the contact line is measured in the image plane $\Pi$ [see Fig. 4(b)] and is related to the width $w$ of the tilted region in the object's plane [see Fig. 4(a)] through the optical system magnification $\gamma=\delta / w$. The width of the triple contact line is given by (see Fig. 4)

$$
t=w \tan \alpha .
$$

Using the relationship (2), we eliminate the tilt angle from (1) and numerically solve the equation for $t$. The displacement of the shadow line $\Delta z$ is significantly larger than the width of the contact line $\delta$ that is sometimes very small, i.e., close to the image resolution limit. To improve the precision, we took 5 to 10 different perpendicular sections of the contact line and determined the average width. Using the average value of the width, we computed the thickness of the film based on formulas (1) and (2). The highest error in the thickness is from the uncertainty in the contact line width $\delta$.

In our system the liquid wets the solid so that the initial state (before heating) is a flattened gas bubble constrained by the two windows and the cell edge. The initial off-center position of the bubble with part of the bubble touching the copper sidewall occurs for experiments A and B and is discussed in detail in Ref. [4]. The cell windows are not exactly parallel and constrain the bubble to press against the sidewall [5].

During these experiments an automatic $T_{c}$ search was performed. The system was first heated to $500 \mathrm{mK}$ above $T_{c}$ and then brought close to $T_{c}$ with quenches of $100,50,25$, and 15 $\mathrm{mK}$. Quenches of $1 \mathrm{mK}$ were then performed past the point of phase separation. The transition temperature was crossed on the very last quench so that the transition temperature is $45.5543{ }^{\circ} \mathrm{C} \pm 0.5 \mathrm{mK}$.

\section{RESULTS AND ANALYSIS}

The initial state of the gas bubble for experiments A and B is shown in Figs. 6(a) (experiment B) and 6(e) (experiment A). Figure 3 shows temperature versus time for the sequence of images shown in Figs. 6 and 7 for experiments A and B. The main difference between experiments A and B is the cell thickness or aspect ratio, given in Table I. The cell used in experiment $\mathrm{A}$ is thicker than the cell used in experiment $\mathrm{B}$ producing a wider meniscus or dark region in the images of 

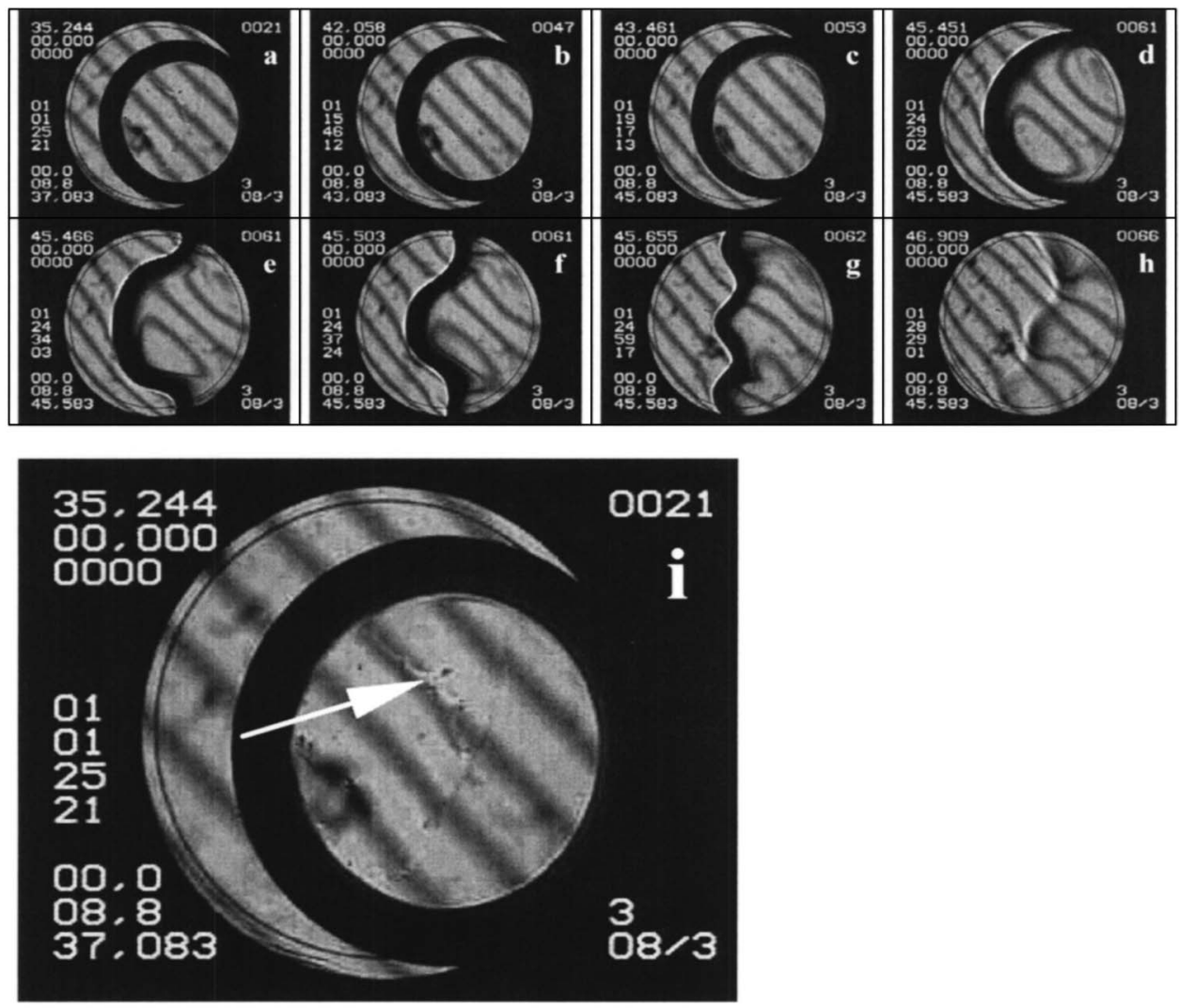

Figs. 6(e)-6(h). In these experiments, the bubble is compressed by the sapphire windows and the slightly inclined windows push the gas bubble to the copper sidewall, forming a bubble sidewall contact region (CR), as discussed in Ref. [4].

Experiment $\mathrm{C}$ was performed in a variable volume cell filled with $\mathrm{CO}_{2}$. The boiling experiments done in this cell yielded quite different results from the boiling runs in experiments A and B. As shown in Ref. [4], the different results also occur at corresponding temperatures in the same fluid $\left(\mathrm{SF}_{6}\right)$, showing that the differences are not a result of the differing media. The different behavior seen in experiment $\mathrm{C}$ is caused by the various constraints placed on the bubble from the presence of the thermistors and the variable volume piston. In fact, these results can be viewed as a control experiment; if the bubble is not free to move and touch the sidewall, then some of the results of experiment A and B do not occur.

When the second ramp commences in experiment $\mathrm{C}$, bubbles begin to nucleate at the sidewall. As can be seen in Fig. 8(a), a cluster of bubbles forms at the edge of the cell, and one of these bubbles has coalesced with the large bubble producing a plume that travels toward the center of the large bubble. The gas released from this coalescence has produced a strong shadowgraph image, i.e., the small bubbles contain hot gas that is pushed into cooler gas, resulting in a changing density gradient. All of this activity produces considerably distorted grid shadow images [Figs. 8(b) and 8(c)].

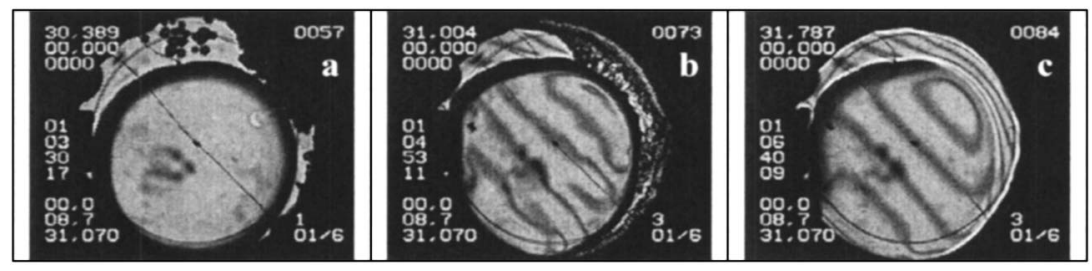

FIG. 8. Shown are some of the events that occurred in the final portion of a ramp similar to the ramps shown in Fig. 3 in a cell filled with $\mathrm{CO}_{2}$. This cell contains three thermistors and a piston to vary the fluid density between ramps. In this case the fluid was at $\delta \rho=-3.5 \%$ below the critical density. Panel (a) shows that a cluster of bubbles has formed at the edge of the cell far below $T_{C}$. One of these bubbles has coalesced with the large bubble producing a hot plume that travels toward the center of the large bubble. Panel (b), at approximately $T_{C}$, shows a considerably distorted grid shadow image with critical opalescence at the sidewall in the region just where the bubbles were nucleating. The dynamical activity suddenly stops at about the same time as this layer forms. The particular phase distribution that existed just before the critical temperature was passed appears to be frozen in place. The image in panel (c) is above the critical temperature $T_{C}$ and the grid shadows in this image shows significant density changes. Slower mass fluxes continue, however, and the system evolves to a uniform supercritical fluid not long after panel (c). 


\section{A. Convection and the isothermal liquid-gas interface}

If a temperature change $\delta T$ appeared along the liquid-gas interface, it would create a surface tension gradient $\delta \sigma$ $=(\mathrm{d} \sigma / \mathrm{d} T) \delta T$ that would drive a thermal-capillary or a Marangoni flow in the bulk of both fluids [10-12]. The possible effects of a surface tension gradient were considered in Ref. [4]. It was shown that such effects would be unlikely to cause large-scale bubble deformations like those in Fig. 7. The reasons were as follows: no convection was observed, the interface is essentially at saturation so that any gradients are weak transients that are damped by evaporation and condensation, and if there were convection it would also dampen any $\delta T$ along the liquid-gas interface [12]. Such a flow, however, could modify the shape of the wetting layer and create large, sustained contact lines. The same reasons for an isothermal bubble interface also apply to the wetting layer. On the other hand, the proximity of the wetting layer to the heating wall could drive such a surface tension gradient. Again, we have not seen any evidence of the steady convection that is required to create and maintain these contact lines in our experiments. We have occasionally seen transient bubble coalescence. These events, however, only had a small transient influence on the bubble shape (a perturbation of less than a second) and no effect on the wetting layer. The fact that there is no convection far from the critical point shows that the interface is isothermal.

\section{B. Vapor recoil and wall drying}

At the beginning of each heating ramp, the grid shadows are not distorted, indicating a flat wetting film. After heating is started, a fast-moving contact line appears near the CR on the right and propagates to the left. On the left side of the contact line [see the arrow in Fig. 2(b)] the grid shadows are distorted while the grid shadows are straight on the right side. We cannot directly detect if there is any liquid present in the undistorted region. If there were appreciable liquid after the propagation of the contact line, we would expect to have significant film thickness distortions. This implies that there is at least a thin, flat liquid film to the right of the contact line.

Figures 6(c) and 6(g) show the thin line with the grid present. Figures 6(b) and 6(f) show the thin line without the grid shadows present. This thin line corresponds to the discontinuity in the grid shadows and produces a thin line by the shadowgraph effect, i.e., the thin line corresponds to a region of strongly changing film thickness. This is the line that we use to identify $\delta$ in the film thickness calculations discussed above. In addition, there are some particles deposited on the sapphire windows, as can be seen in Figs. 6(i) and 7(i). There are two thin lines with one line on each window, and these particles have distorted one of the thin lines. This strongly suggests that these thin lines are liquid-gas-solid contact lines in that they can be pinned by a defect while advancing or receding. The constraint of zero actual contact angles, however, means that these are not the usual triple contact lines that occur on a partially wetted solid. Because a rapid change in curvature occurs near the sapphire wall, we can still consider this as a contact line if the usual contact angle at the solid-liquid-gas interface is replaced by an apparent contact angle. On a larger scale, the region of large curvature between straight sections of interface appears as a cusp that the apparent contact angle characterizes.

In experiments $\mathrm{A}$ and $\mathrm{B}$, the gas is in contact with the copper sidewall during heating as implied by Fig. 6, Fig. 7, and by the numerical calculation of the initial bubble shape in Ref. [4]. Garrabos et al. observed a striking spreading bubble process that occurs closer to $T_{\mathrm{CX}}$ [4]. An example of this spreading is shown in Fig. 7 (Fig. 8 in Ref. [4]). They reasoned that a vapor recoil force that diverges strongly at $T_{c}$ was pushing the bubble interface. The vapor recoil (also called vapor thrust) is the only effect that we can find that could cause such a large mechanical response. In this process, the bubble is deformed through evaporation, i.e., by the normal stress exerted on the interface by the recoil from departing vapor $[13,14]$. The vapor recoil force is large in the vicinity of a triple contact line [14]. This can be seen qualitatively by considering that the liquid-gas interface temperature is fixed by the saturation condition, whereas the wall's temperature is larger. Near the triple contact line, where these two surfaces converge, a large temperature gradient forms and the largest portion of mass transfer also takes place. As noted in Ref. [4], other experiments in the same media where the bubble is not pushed against the sidewall show no bubble spreading. In fact, as shown in Fig. 8, small bubbles nucleated near the sidewall in experiment $\mathrm{C}$ when the large bubble was near the sidewall. This region also had many coalescence events and a considerable density gradient. Similar coalescence events were also seen close to the $\mathrm{CR}$ in experiment $\mathrm{B}$ before the rapid bubble spreading appeared. This transient phenomenon stops prior to the rapid spreading, indicating that the CR has grown and the wetting film has evaporated along the copper sidewall. In fact, in experiments $\mathrm{A}$ and $\mathrm{B}$ we see no interface near the $\mathrm{CR}$, and the grid shadows near the sidewall do not show the type of displacements that they do in Fig. 8. We conclude that the sidewall near the $\mathrm{CR}$ is dry.

By the same reasoning as above, we conclude that the vapor recoil force also causes the receding contact line. This is supported by the observations, especially in experiment A, where the bubble initially responds to the heating with a small impulse and a receding contact line [see Fig. 2(b)]. Because the heat flow impedance of the copper wall is lower than the sapphire windows, we expect a stronger evaporation near the CR, i.e., this hotter region would tend to quickly evaporate any thin film to the right of the contact line. The evaporation also makes the region behind the triple contact line become dry. We therefore identify the thin line as a triple contact line and the area to the right of the contact line as the dry area. The evaporation of the wetting film between the sapphire windows and the gas bubble leads to the moving contact line (see Fig. 4). It follows that the copper sidewall also dries prior to the drying of the less thermally conductive window.

\section{Moving contact lines in heated liquid film}

Less than $30 \mathrm{~s}$ after the heating begins, the speed of the rapidly moving contact line decreases to almost zero, as 

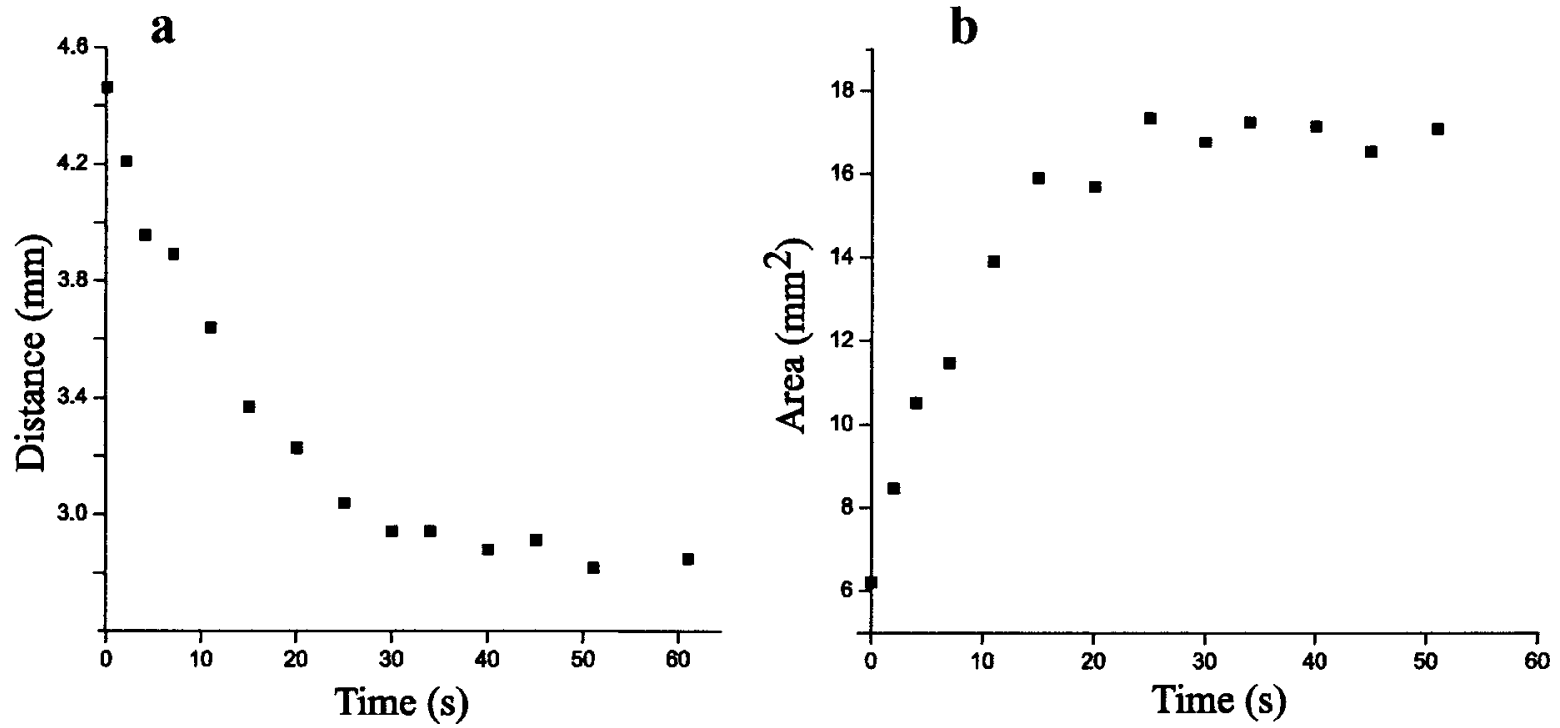

FIG. 9. (a) The position of the contact line with respect to the center of the cell versus time (see Fig. 2) after heating begins. (b) The dry area behind the triple contact line and the interior of the dark meniscus region rapidly increases while the contact line moves toward the cell's center.

shown in Fig. 9(a). After this fast transient motion the contact line appears stationary. Over the longer time scale of the entire ramp we observed a slow evolution. We now discuss these two different regimes of the triple contact line movement.

\section{Fast moving contact line}

At the beginning of both temperature ramps (after the heating starts) and intermittently during the ramp, a fastmoving contact line is seen. This triple contact line slows as it recedes toward the cell's center (Fig. 9). This rapid increase of the dry area behind the contact line is the result of the fast evaporation that takes place first at the copper wall and spreads to the sapphire surface. A quantitative measure of the change in the dry area is the speed of the contact line. To determine this speed we draw a radius from the cell center perpendicular to the triple contact line (see Fig. 2). By measuring successive intersections of the triple contact line with the reference radius, we were able to determine the normal velocity of the contact line. The measurements in Fig. 9 show that the triple contact line moves rapidly for approximately $20 \mathrm{~s}$ and after almost $30 \mathrm{~s}$ stops. We linearly interpolate between the initial six points (first $20 \mathrm{~s}$ ) to find the average velocity of this initial fast-moving contact line.

We can understand this behavior as follows. Once the gas makes significant contact with the cooler sapphire window the evaporation rate decreases and the speed of the contact line decreases until a steady-state regime is reached. Using the wedge model we determined the wetting film thickness (see the Appendix) at the position shown in Fig. 2(b). The wetting film thickness versus time at this position is plotted in Fig. 10(a). Assuming that the liquid film has a steady shape as the contact line moves, Fig. 10(b) shows the film profile near the contact line as measured from the heating wall towards the center of the cell [see Fig. 2(b)]. As shown in the image data, the film thickness is relatively constant farther from the contact line. During the fast receding of the contact line, it appears that the force acting near the wall pushes the fluid to the right, where it accumulates and creates a rim. A rim such as this is quite common in dewetting phenomena [16].

\section{Slow dynamics of the contact line}

After the initial fast motion, the contact line (and the dry area) changes at a much slower rate, eventually stopping altogether. Calculations of the film thickness corresponding to these slowly moving and stationary contact lines over the entire ramping time (about $100 \mathrm{~min}$ ) are shown in Fig. 11. The film thickness estimates are based on the same wedge model of the interface used for the fast-moving contact line. We have also observed significant fluctuations in the film thickness in the slow regime.

The area of the gas bubble is essentially constant during the heating ramp [Figs. 12(a) and 12(b)]. On the other hand, the relative surface dry area, as shown in Figs. 12(c) and 12(d), has a more complicated behavior. In the first part of a ramp, we observed a decrease in the dry area [Figs. 12(c) and 12(d)], whereas in the second part of a ramp toward $T_{C}$ the dry area stabilizes to a constant value for a large range of the relative temperature [Fig. 12(d)].

Figure 12 shows that the gas bubble area (or volume) stays constant while the dry area changes. There is also a strong correlation between the estimated film thicknesses in Fig. 11(a) and the total film surface area (Fig. 12). This shows that the liquid film tends to conserve mass over the long time scale. The steady state is also accompanied by strong fluctuations in the film thickness after the initial fast advance. These fluctuations show that this steady state is a delicate balance between heat transfer, mass transfer, and mechanical stress. 

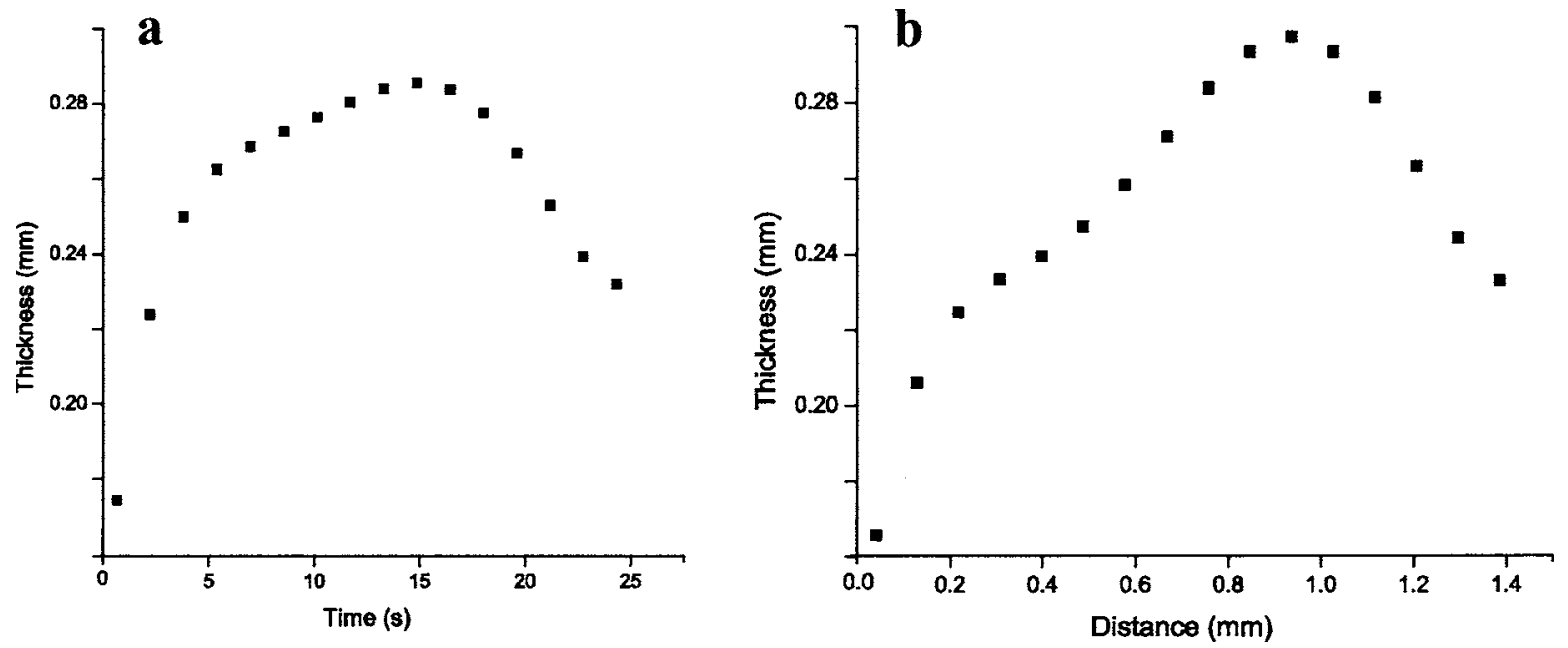

FIG. 10. The thickness of the wetting film near the triple contact line was computed using the wedge model at the position shown by the point in Fig. 2(b) (data from experiment B). (a) Estimated thickness of the wetting film versus time calculated at the beginning of the second heating ramp with respect to cell's center. (b) Estimated spatial profile of the contact line based on the relationship between the distance swept by the contact line and the elapsed time.

\section{Close to $T_{\mathrm{CX}}$}

As $T$ approaches $T_{\mathrm{CX}}$, the contact line disappears (the last contact line is seen at $\approx 44.4^{\circ} \mathrm{C}$ in experiments $\mathrm{A}$ and $\mathrm{B}$ ) and the density gradient near the liquid-gas interface increases (see Fig. 7). Small impulsive perturbations are also intermittently seen near the CR. These impulses perturb the liquidgas interface, with the interface returning to its original shape after less than a second. Closer to $T_{\mathrm{CX}}$ the bubble spreads along the copper sidewall, as discussed in Ref. [4] [also see Figs. $7(\mathrm{e})-7(\mathrm{~g})]$. At $T \approx T_{\mathrm{CX}}$, the interface becomes a density gradient [Fig. 7(h)].

When very close to the critical point the vapor bubble's behavior becomes quite complex, and the grid shadows show large displacements near the interface. In experiment $\mathrm{C}$, as shown in Fig. 8, the isolated bubbles actively coalesce at lower temperature. This coalescence activity rapidly slows near $T_{\mathrm{CX}}$ and the bubbles "freeze," i.e., they remain motionless very near $T_{\mathrm{CX}}$ in what may be described as a mechanical slowing down. In addition, the large bubble shows regions of nonuniform curvature [see, e.g., Fig. $7(\mathrm{~g})]$. In experiments $\mathrm{A}$ and $\mathrm{B}$ the bubble rapidly recedes over the sidewall [see Figs. 7(e)-7(h)]. As shown below, convective transport of heat cannot rapidly equilibrate the interface temperature. The other modes of heat transport are also very inefficient as $T \rightarrow T_{C}$, i.e., temperature diffusion becomes very slow as the thermal diffusivity goes to zero, $D_{\text {th }} \sim\left(T_{C}-T\right)^{0.85} \rightarrow 0$ [15] and the latent heat also goes to zero as $\left(T_{C}-T\right)^{\beta}$, where $\beta=0.325$ [17]. In our experiment, however, heating continues as the cell's temperature is ramped past $T_{C}$ at the same constant rate. The probability of a temperature change $\delta T(x)$ along the interface (where $\boldsymbol{x}$ is a position at the interface) increases close to $T_{C}$.
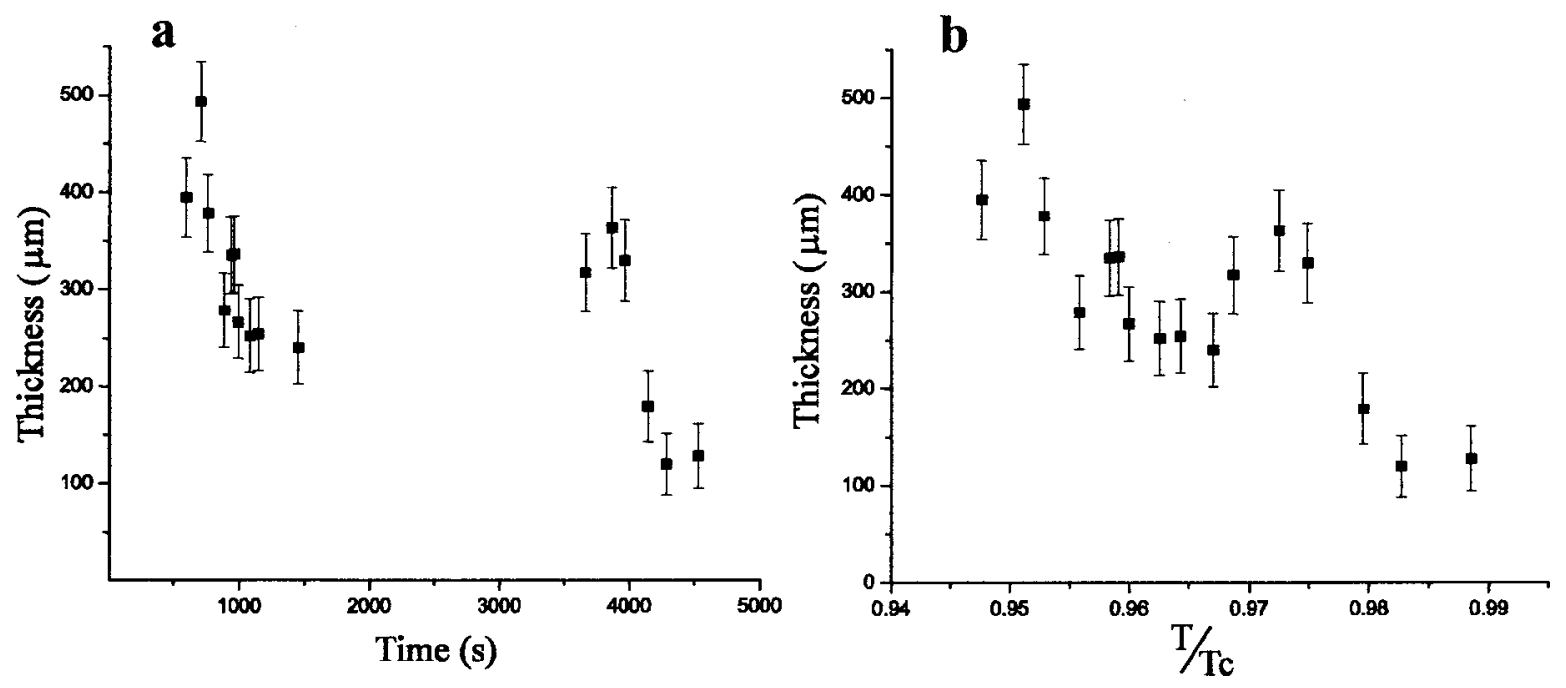

FIG. 11. Estimated thickness of the wetting film versus time (a) and versus relative temperature (b). The data refer to experiment B and used the linear wedge model. The error bars reflect the uncertainty in the measurements of the contact linewidth. 

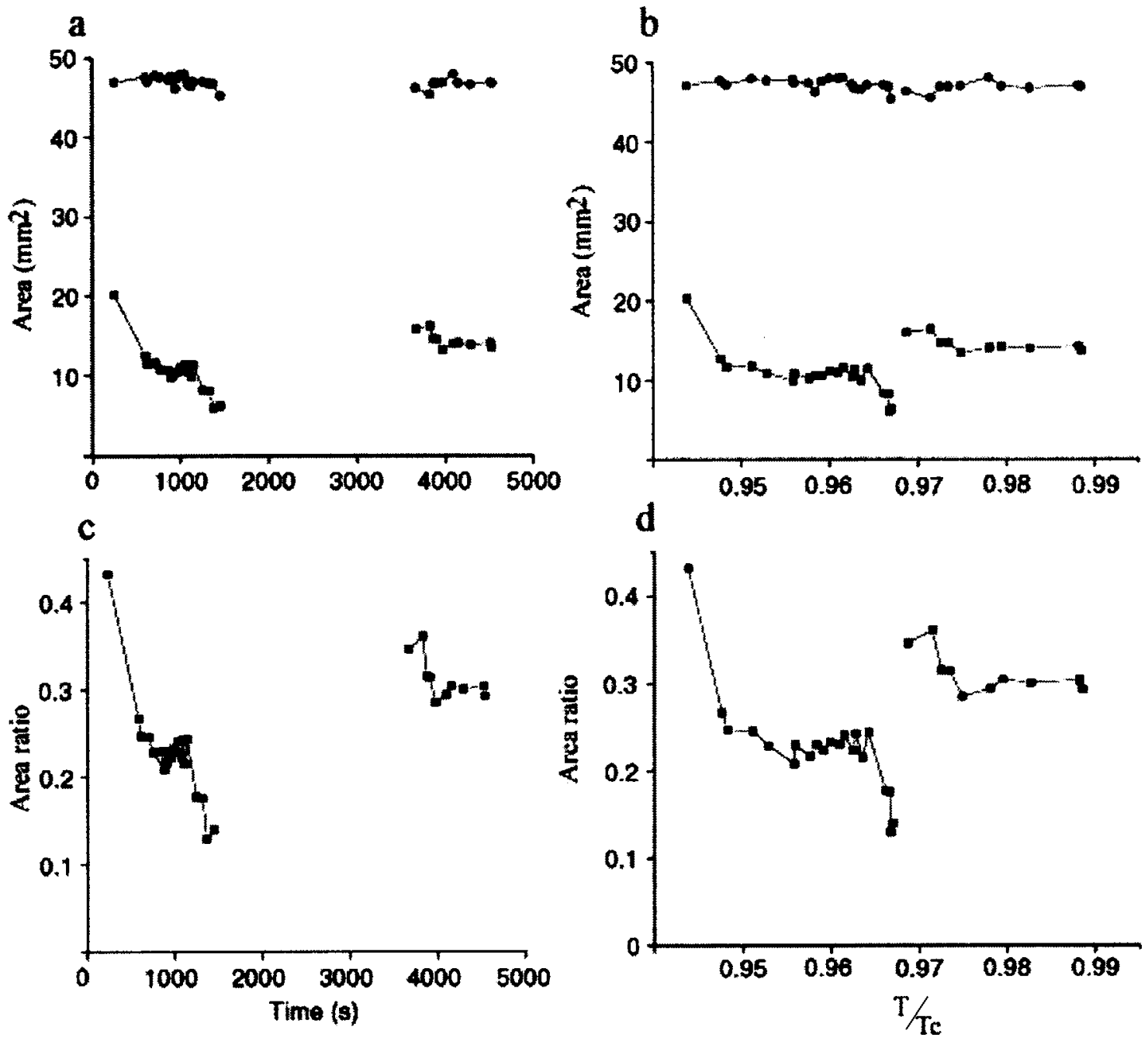

FIG. 12. Dry area [squares in panels (a) and (b)] and the total area of the gas bubble [circles in panels (a) and (b)] versus time [panel (a)] and relative temperature [panel (b)]. The data are from experiment B and both areas were measured with respect to the interior of the dark meniscus. The total area of the gas bubble is constant during heating [circles in panels (a) and (b)]. The ratio of the dry area, behind the contact line, to the total area of the gas bubble, versus time [panel (c)], and versus the relative temperature [panel (d)] is shown.

The dimensionless parameter that governs the stability of an isothermal interface subjected to a temperature gradient normal to the interface is the Marangoni number, given by $\mathrm{Ma}=\delta \sigma l /\left(\eta D_{\mathrm{th}}\right)$, where $l$ is the characteristic length that $\sigma$ varies by $\delta \sigma$ and $\eta$ is the viscosity. This number is the ratio of the surface tension driving force to the viscous dissipation force, so that if this number is larger than $\sim 100$ a stationary interface becomes unstable to surface tension-driven flow [18]. Close to the critical point the surface tension vanishes with the universal form $\sigma=\sigma_{o}\left(T_{C}-T\right)^{2 \nu}$, where $\nu=0.63$ is a universal exponent. This implies that $\delta \sigma=-2 v \sigma_{o}\left(T_{C}\right.$ $-T)^{2 \nu-1} \delta T(\boldsymbol{x}) \sim\left(T_{C}-T\right)^{0.26} \delta T(\boldsymbol{x})$. This factor and the $D_{\text {th }}$ factor gives Ma the form $\mathrm{Ma} \sim\left(T_{C}-T\right)^{-0.59}$ that diverges as $T \rightarrow T_{C}$. Any surface tension-driven convection, however, has a characteristic velocity measured by $U \sim \delta \sigma / \eta$, where we assume that the viscous stress balances the surface tension gradient. This velocity goes to zero near $T_{C}$ as $U \sim\left(T_{C}\right.$ $-T)^{0.26}$. Very close to the critical point, the velocity of the flow produced by a possible $\delta T(\boldsymbol{x})$ is probably too small to observe, and would tend to equilibrate the interface at a slow rate. As shown in Ref. [4], this system is near saturation and therefore lacks strong thermal forcing at the interface from evaporation. If this flow exists, it would probably be a weak transient effect as the convection drives the interface to a uniform temperature.

Close to the critical point any slow transient flow from a surface tension change, together with weak latent heat and thermal diffusivity, could leave a corresponding temperature change $\delta T(x)$ at the interface for a considerable time. This temperature change $\delta T(x)$ could change the bubble's shape by inducing a surface tension variation $\delta \sigma(x)$ on the interface in a manner analogous to a nonuniform elastic membrane. We now analyze this possibility and compare its consequences with our data.

Because pressure changes relax much faster than temperature changes, on a long time scale the bubble may deform with the pressure remaining uniform. Assuming again that the viscous stress balances the surface tension gradient in the direction tangent to the interface, we may then use Laplace's formula for the normal direction. This implies that the local curvature $c(x)$ and the local surface tension 
are inversely proportional, i.e., $p=\sigma(\boldsymbol{x}) c(\boldsymbol{x})=$ constant for a steady interface where all quantities with local variations are written as functions of $\boldsymbol{x}$. As $T \rightarrow T_{C}$, it is clear that $p \rightarrow 0$ and $\sigma(x) \rightarrow 0$. There is still the question, however, of the behavior of $c(\boldsymbol{x})$ and the shape of the interface that may either: (i) diverge (e.g., the radius of a spherical bubble goes to zero or a finite interface becomes unstable); (ii) converge to a constant (bubble shape distorts or stays the same); or (iii) converge to zero (bubble interface becomes flat). It is highly likely that some of the small, hot bubbles that nucleated at the walls in experiment $\mathrm{C}$, shown in Fig. 8, would have had a $\delta T(\boldsymbol{x})$ and a curvature divergence. Instead of observing a destabilizing curvature divergence we observed the mechanical slowing down ruling out case (i). Case (iii) may also be ruled out because $c(x) \rightarrow 0$ implies that the interface becomes flat, whereas we observe the mechanical slowing down without any bubbles flattening out. This leaves case (ii), where $c(\boldsymbol{x}) \rightarrow c_{0}=$ const as $T \rightarrow T_{C}$. This is what is observed in the mechanical slowing down, where we see the sharp interface replaced by a diffuse density gradient with no mechanical forcing. The bubbles may deform under a $\delta T(\boldsymbol{x})$ perturbation, but do so such that they keep the same constant curvature as $T \rightarrow T_{C}$. Alternatively, an interface may not have a $\delta T(x)$ perturbation, and the bubbles keep the same shape as $T \rightarrow T_{C}$.

The spreading bubble in Figs. $7(\mathrm{e})-7(\mathrm{~g})$ shows a change of curvature that seems large. The majority of curvature at the interface, however, is still in a plane perpendicular to the field of view, as can be seen by the dark, thick line. This large curvature may easily compensate for the curvature changes that are seen in the field of view as $T \rightarrow T_{C}$. If the spreading bubble was caused by a $\delta T(\boldsymbol{x})$ perturbation, we would expect to see similar deformations in the small bubbles of Fig. 8 that are clearly hotter than the ambient fluid [see the plume in Fig. 8(a)]. As pointed out in Ref. [4], however, the vapor recoil force is strongly divergent as $T \rightarrow T_{C}$. The interface shapes in Figs. 7(e)-7(h) are more likely the result of the slow response of the soft interface $(\sigma \rightarrow 0)$ to the rapid spreading of the bubble at the copper sidewall. The interface is simply too soft and slow to relax to equilibrium before the temperature arrives at $T_{C}$. At $T \geqslant T_{C}$ the bubble's relaxation from surface tension is negligible, and the "interface" shape is defined by local mass fluxes.

\section{CONCLUSION}

We have reported and interpreted phenomena observed as a two-phase fluid's temperature is ramped past its critical temperature (near-critical boiling) in a weightless environment. We observed moving contact lines and a spreading bubble in several $\mathrm{SF}_{6}$ cells where the bubble touched the copper sidewalls. We also used ray tracing and the defocused grid technique to estimate the film thickness near the contact lines using a wedge model. The fast motion of the contact line appears to accumulate fluid in front of the line, and the steady-state line exhibits thickness fluctuations. We observed a rapid bubble spreading that was previously reported and explained using the vapor recoil force. We were also able to interpret our results using this force. We showed that in the presence of a local temperature change $\delta T(x)$ the interface curvature stays constant very close to $T_{C}$. We conclude that the large bubble's unusual shape after the spreading is the result of the slow response of the soft interface to the rapidly receding contact line at the copper sidewall. The quantitative detail of the heat and mass transfer processes near the triple contact line is a challenging problem that exceeds the scope of this study. The qualitative explanation for the dynamics of the contact line is that the vapor recoil force exerts a stress on the liquid-gas interface near the contact line.

\section{ACKNOWLEDGMENTS}

We gratefully acknowledge the support of CNES and NASA through NASA-OBPS Grants NAG3-1915 and NAG3-2447. We thank all of the Alice II team, especially J. F. Zwilling, and all individuals involved in the Mir missions for their technical support.

\section{APPENDIX}

To simplify the analysis, we assume that only one liquidgas interface is tilted (Fig. 5) and that the tilt angle is $\alpha$. Let $z_{0}$ be the height of an incident ray parallel to the optical axes ( $x$ axis). At point $I_{1}$ Snell's law gives

$$
n_{g} \sin \alpha=n \sin r_{1},
$$

where $n_{g}$ is the index of refraction of the gas, $n_{l}$ is the index of refraction of the liquid, and $r_{1}$ is the refraction angle at $I_{1}$. Applying Snell's law at the point $I_{2}$, we get

$$
n_{l} \sin \left(\alpha-r_{1}\right)=n_{s} \sin r_{2},
$$

where $n_{s}$ is the sapphire index of refraction and $r_{2}$ is the refraction angle at $I_{2}$. The height of the next incidence point $\left(I_{2}\right)$ is

$$
z_{2}=z_{0}-t \tan \left(\alpha-r_{1}\right) .
$$

Finally, there is another refraction inside the cell

$$
n_{s} \sin r_{2}=n_{\text {air }} \sin r_{3},
$$

where $r_{3}$ is the angle of the emergent ray and its distance from the optical axes is

$$
z_{3}=z_{2}-H_{s} \tan r_{2}=z_{0}-t \tan \left(\alpha-r_{1}\right)-H_{s} \tan r_{2},
$$

where $H_{s}$ is the thickness of the sapphire window. The emergent ray from the cell hits the convergent lens at $z_{4}$, which is given by

$$
z_{4}=z_{3}-x_{1} \tan r_{3}=z_{0}-t \tan \left(\alpha-r_{1}\right)-H_{s} \tan r_{2}-x_{1} \tan r_{3} .
$$

Using congruent triangles, we get the height of the point in the CCD plane where the refracted ray $I_{4} I_{5}$ hits the plane (point $I_{6}$ )

$$
\frac{z_{6}-\delta}{z_{4}+\delta}=\frac{x_{2}-f}{f} \Rightarrow z_{6}=\delta+\left(z_{4}+\delta\right) \frac{x_{2}-f}{f},
$$

where $\delta=f \tan r_{3}$ is the height of the crossing point between the refracted ray $I_{4} I_{5}$ and the focal plane. Based on (A6) and 
(A7), we get the displaced height of the grid shadow at

$$
\begin{aligned}
z_{6}= & {\left[z_{0}-t \tan \left(\alpha-r_{1}\right)-H_{s} \tan r_{2}-x_{1} \tan r_{3}+f \tan r_{3}\right] \gamma } \\
& +f \tan r_{3},
\end{aligned}
$$

where $\gamma=x_{2} / x_{1}$ is the magnification of the convergent lens $L$. Based on (A7), the height of the crossing point between the unperturbed ray and the CCD plane will be

$$
z_{6}^{0}=z_{0} \gamma
$$

Combining the above relationships, we get the displacement of the grid shadow due to a tilted liquid-gas interface

$$
\Delta \mathrm{z}(t, \alpha)=-\left[t \tan \left(\alpha-r_{1}\right)+H_{s} \tan r_{2}\right] \gamma .
$$

The displacement of the grid shadow depends on both the tilt angle $\alpha$ and the thickness of the film $t$. Based on experimental data such as those shown in Fig. 4, we can measure

(i) the displacement of the grid line shadows $\Delta z(t, \alpha)$, and

(ii) the width of the triple contact line $w$.

The width of the triple contact line is given by (Fig. 5)

$$
t=w \tan \alpha .
$$

Based on (A1), (A2), (A10), and (A11), we are able to determine the tilt angle $\alpha$ of the liquid-gas interface and the thickness of the wetting film.
[1] L. S. Tong, Boiling Heat transfer and two-phase flow (Taylor and Francis, New York, 1997).

[2] M. R. Moldover, J. V. Sengers, R. W. Gammon, and R. J. Hoken, Rev. Mod. Phys. 51, 79 (1979).

[3] P. G. De Gennes, Rev. Mod. Phys. 57, 827 (1985).

[4] Y. Garrabos, C. Lecoutre-Chabot, J. Hegseth, V. S. Nikolayev, D. Beysens, and J. Delville, Phys. Rev. E 64, 051602 (2001).

[5] J Hegseth, Y. Garrabos, V. S. Nikolayev, C. Lecoutre-Chabot, R. Wunenburger, and D. Beysens, Int. J. Thermophys. 23, 89 (2002).

[6] C. Morteau, M. Salzman, Y. Garrabos, and D. Beysens, in Proceedings of the 2nd European Symposium on Fluids in Space, edited by A. Viviani (Congressi SRL, Rome, 1997), p.327.

[7] J. P. Delville, C. Salzman, Y. Garrabos, and D. Beysens, in Ref. [6], p. 312.

[8] V. Gurfein, D. Beysens, Y. Garrabos, and B. Le Neindre, Opt. Commun. 85, 147 (1991).

[9] A. Oprisan, thesis, New Orleans University, 2003.

[10] S. H. Davis, Annu. Rev. Fluid Mech. 19, 403 (1987).
[11] J. R. A. Pearson, J. Fluid Mech. 4, 489 (1958).

[12] J. J. Hegseth, N. Rashidnia, and A. Chai, Phys. Rev. E 54, 1640 (1996).

[13] H. J. Palmer, J. Fluid Mech. 75, 487 (1976).

[14] V. S. Nikolayev and D. Beysens, Europhys. Lett. 47, 345 (1999).

[15] J. Straub, in Proceedings of the IXth European Symposium on Gravity-Dependent Phenomena in Physical Sciences, edited by L. Rathe, H. Walter, and B. Feuerbacher (Springer, Berlin, 1995), p. 351.

[16] C. Andrieu, C. Sykes, and F. Brochard, J. Adhes. 58, 15 (1996).

[17] By the Clausius-Clayron equation, the latent heat is proportional to the density difference and the later goes to zero as $\left(T_{C}-T\right)^{\beta}$. See M. Zemanski and R. H. Dittman, Heat and Thermodynamcis (McGraw-Hill, New York, 1997), pp. 286289.

[18] M. F. Schatz, S. J. VanHook, W. D. McCormick, J. B. Swift, and H. L. Swinney, Phys. Rev. Lett. 751938 (1995). 